\title{
MicroRNA clusters: dysregulation in lung adenocarcinoma and COPD
}

\author{
Sonia Molina-Pineloํ․ M. Dolores Pastor ${ }^{1}$, Rocío Suarez¹, \\ Beatriz Romero-Romero², Miriam González De la Peña³, Ana Salinas', \\ Rocío García-Carbonero ${ }^{1,3}$, María José De Miguel ${ }^{3}$, Francisco Rodríguez-Panadero, \\ Amancio Carnero ${ }^{1}$ and Luis Paz-Ares ${ }^{1,3}$
}

Affiliations: 'Instituto de Biomedicina de Sevilla (IBIS), HUVR, CSIC, Universidad de Sevilla, Seville, Spain. ${ }^{2}$ Pneumology Dept, Hospital Universitario Virgen del Rocío, Seville, Spain. ${ }^{3}$ Medical Oncology Dept, Hospital Universitario Virgen del Rocío, Seville, Spain.

Correspondence: Luis Paz-Ares, Medical Oncology Dept, Hospital Universitario Virgen del Rocío, Manuel Siurot s/n. 41013 Seville, Spain. E-mail: Ipazaresahotmail.com

ABSTRACT Lung adenocarcinoma and chronic obstructive pulmonary disease (COPD) are pulmonary diseases that share common aetiological factors (tobacco smoking) and probable dysregulated pathways. MicroRNAs (miRNAs) play an essential role in regulating numerous physiological and pathological processes. The purpose of this study was to assess global miRNA expression patterns in patients with COPD and/or adenocarcinoma to elucidate distinct regulatory networks involved in the pathogenesis of these two smoking-related diseases.

Expression of 381 miRNAs was quantified by TaqMan Human MicroRNA A Array v2.0 in bronchoalveolar lavage fluid samples from 87 patients classified into four groups: COPD, adenocarcinoma, adenocarcinoma with COPD, and control (neither COPD nor adenocarcinoma). 11 differentially expressed miRNAs were randomly selected for validation in an independent cohort of 40 patients.

Distinct miRNA expression profiles were identified and validated for each pathological group, involving 66 differentially expressed miRNAs. Four miRNA clusters (the mir-17-92 cluster and its paralogues, mir-106a-363 and mir-106b-25; and the miR-192-194 cluster) were upregulated in patients with adenocarcinoma and one miRNA cluster (miR-132-212) was upregulated in patients with COPD.

These results contribute to unravelling miRNA-controlled networks involved in the pathogenesis of adenocarcinoma and COPD, and provide new tools of potential use as biomarkers for diagnosis and/or therapeutic purposes.

@ERSpublications

MicroRNA expression profiles in bronchoalveolar lavage fluid enable discrimination of adenocarcinoma from COPD http://ow.ly/tPaVC

\footnotetext{
This article has supplementary material available from www.erj.ersjournals.com

Received: May 292013 | Accepted after revision: Feb 142014 | First published online: April 172014

Support statement: L. Paz-Ares is funded by Fondo de Investigación Sanitaria (PI1102688) and RTICC (R12/0036/0028). S. Molina-Pinelo is funded by Fondo de Investigación Sanitaria (CD1100153), Fundación Científica de la Asociación Española Contra el Cáncer, Consejería de Salud y Bienestar Social PI-0046-2012 and Fundación Mutua Madrileña (2010). M.D. Pastor is funded by Fondo de Investigación Sanitaria (CD0900148). The A. Carnero laboratory was supported by grants to from the Spanish Ministry of Economy and Competitivity, ISCIII (PI12/00137, RTICC: RD12/0036/0028), Consejeria de Ciencia e Innovacion (CTS-6844) and Consejeria de Salud of the Junta de Andalucia (PI-0135-2010 and PI-0306-2012).
}

Conflict of interest: None declared.

Copyright @ERS 2014 


\section{Introduction}

Lung cancer is the leading cause of cancer-related death worldwide. Tobacco exposure continues to be the main risk factor for lung cancer and is also closely linked to the pathogenesis of chronic obstructive pulmonary disease (COPD) [1]. By contrast, COPD increases the risk of developing lung cancer independent of tobacco use. In fact, $50-80 \%$ of patients with lung cancer have COPD [1]. More specifically, squamous cell carcinoma followed by adenocarcinoma are the most common histological subtypes among patients with COPD and among long-term smokers [2, 3]. Lung cancer and COPD are, therefore, pulmonary diseases that share common aetiological factors and likely pathogenic mechanisms. Consistent with this, Young et al. [4] have provided evidence that lung cancer and COPD are directly linked at a molecular genetic level.

The discovery of microRNAs (miRNAs) in the early 1990s revealed an unexpected level of gene expression regulation that has proven to be of great relevance in the regulation of numerous physiological and pathological conditions, including carcinogenesis, cancer progression and response to therapy [5]. miRNAs are small, noncoding RNAs that function at a transcriptional and post-transcriptional level, usually resulting in gene silencing via mRNA translational repression or target degradation. Overexpressed miRNAs may function as oncogenes by negatively regulating tumour suppressor genes and/or genes that control cell differentiation or apoptosis, and underexpressed miRNAs may act as tumour suppressor genes if their target genes are oncogenes [6]. Moreover, increasing evidence supports the potential role of miRNAs as diseasespecific biomarkers, generating promising new tools for diagnostic, preventive or therapeutic purposes. The relevance of miRNA-mediated regulation is further underscored by the fact that miRNA genes tend to cluster throughout the genome, and this clustering is highly conserved among species [7]. Some miRNAs are, therefore, transcribed from polycistronic primary transcripts, each of which may act independently or within a coordinated regulatory network. In lung cancer, miRNA profiling has been proposed as a useful aid in the diagnosis, prediction of recurrence and assessment of a patient's prognosis in different clinical scenarios $[8,9]$. miRNA clusters have also been recognised as essential components of known lung cancer pathways, as is the case for the miR-17-92 cluster in small cell lung cancer, which regulates growthstimulatory signalling pathways [10].

Improved understanding of the molecular networks controlled by miRNAs may provide new tools of potential use as biomarkers for screening, diagnosis and/or therapeutic targeting. Based on these premises, the purpose of this study was to assess miRNA expression patterns, in cooperation or independently, and their molecular targets in patients with COPD and/or adenocarcinoma to elucidate distinct regulatory patterns involved in the pathogenesis of these two smoking-related diseases.

\section{Materials and methods}

\section{Patients and clinical specimens}

miRNA signatures were assessed in the cell fraction of bronchoalveolar lavage (BAL) samples obtained from 87 prospective patients undergoing diagnostic bronchoscopy at the Virgen del Rocío University Hospital, Seville, Spain (training cohort). The expression of randomly selected miRNAs was validated in an independent cohort of 40 patients (validation cohort). All patients were required to meet the following selection criteria: 1) be under pneumology consultation for haemoptysis or pulmonary nodule; 2) be smokers or ex-smokers with $>20$ pack-years of exposure; and 3) be $>40$ years of age. The exclusion criteria for study entry were: 1) diagnosis of a neoplastic disease other than lung adenocarcinoma; 2) active pulmonary tuberculosis or any other active infection; 3) previous lung resection; 4) history of drug abuse; and 5) presence of other acute or chronic inflammatory disease other than COPD. All patients underwent fine-needle or core biopsy for lung cancer diagnosis and none of them had undergone surgery at the time at which the BAL study sample was obtained. Patients were classified according to medical history and spirometric assessment into four groups: a COPD group, a COPD-adenocarcinoma group, an adenocarcinoma group and a control group with neither COPD nor adenocarcinoma. The study protocol was approved by the Institutional Ethics Committee (Virgen del Rocío University Hospital, Seville, Spain) and a written informed consent was obtained from all patients prior to study entry.

\section{miRNA expression profiling}

BAL fluid samples were obtained and processed as previously described [10]. Total RNA, containing small RNA, was extracted from the cell fraction of BAL samples and quantified as previously described [11]. Expression of 381 human miRNAs was quantified in the training cohort using the TaqMan Human MicroRNA A Array v2.0 (Applied Biosystems (Life Technologies S.A.), Madrid, Spain) according to the manufacturer's instructions. 


\section{miRNA expression validation}

Expression of 11 randomly selected miRNAs was validated in an independent cohort of 40 patients using the specific TaqMan MicroRNA assays according to the manufacturer's instructions. Briefly, $2 \mathrm{ng} \cdot \mu \mathrm{L}^{-1}$ total RNA was used to make cDNA by a reverse transcriptase reaction that was performed by sequential incubation at $16^{\circ} \mathrm{C}$ for $30 \mathrm{~min}, 42^{\circ} \mathrm{C}$ for $30 \mathrm{~min}$ and $85^{\circ} \mathrm{C}$ for $5 \mathrm{~min}$. PCR reaction mixture $(10 \mu \mathrm{L})$ contained $0.66 \mu \mathrm{L}$ reverse transcriptase reaction product, $5 \mu \mathrm{L}$ TaqMan $2 \times$ Universal PCR Master Mix and $0.5 \mu \mathrm{L}$ of the appropriate TaqMan MicroRNA Assay $(20 \times)$ containing primers and probe for the miRNA of interest (Applied Biosystems). The mixture was initially incubated at $95^{\circ} \mathrm{C}$ for $10 \mathrm{~min}$, followed by 40 cycles at $95^{\circ} \mathrm{C}$ for $15 \mathrm{~s}$ and $60^{\circ} \mathrm{C}$ for $60 \mathrm{~s}$.

\section{Statistical analysis}

Descriptive statistics were used to characterise the most relevant clinical parameters. The association of categorical variables was explored by the Chi-squared test or Fisher's exact test. To assess distribution of continuous variables among the study groups parametric (t-test) or nonparametric tests (Kruskal-Wallis or Mann-Whitney tests) were employed when appropriate. Statistical analysis was performed using the Statistical Package for the Social Sciences software (SPSS 17; SPSS Inc., Chicago, IL, USA).

Expression of target miRNAs was normalised to the expression of MammU6, which was found in the literature to be the most widely used endogenous miRNA control for quantitative RT-PCR. One nonhuman miRNA was used in each experiment as a negative control. Finally, the results were processed and analysed on an ABIPrism 7900 HT Sequence Detection System (Applied Biosystems). Cycle threshold (Ct) values were calculated with the SDS software v.2.3 (Applied Biosystems) using automatic baseline settings and a threshold of 0.2. Relative quantification of miRNA expression was calculated by the $2^{-\Delta \mathrm{Ct}}$ method [12]. The raw and normalised TaqMan array data have been deposited in the Gene Expression Omnibus under the accession number GSE44531. miRNAs expression was computed using RealTime StatMiner software v.4.2 (Integromics, Inc., Armilla, Spain). This software performs a moderate t-test between groups (pathological groups versus control group) and corrects them using the Benjamini-Hochberg algorithm with the false discovery rate set at a lower value of 0.3 . For undetected miRNAs with $\mathrm{Ct}$ values beyond the maximum $\mathrm{Ct}$ of 32, the StatMiner software inputs a value set to the maximum Ct. To identify direct mRNA targets of miRNAs, we applied three algorithms: miRanda [13], TargetScan release 6.0 [14], and miRWalk [15].

\section{Results}

The main clinical and pathological characteristics of the training $(n=87$ patients $)$ and validation $(n=40$ patients) cohorts did not substantially differ and are summarised in tables 1 and 2 . As expected, $>80 \%$ of patients in each cohort were male. Within each pathological condition, groups were comparable with respect to COPD severity and/or lung cancer histology. In addition, miRNA expression levels did not significantly differ between current and former smokers (data not shown). Both cohorts included a control group of patients with neither COPD nor adenocarcinoma.

We identified discriminating miRNA signatures for each pathological entity (adenocarcinoma, COPD and adenocarcinoma with COPD) (fig. 1), involving 66 differentially expressed miRNAs as compared to the control group $(\mathrm{p}<0.05) .40$ miRNAs were upregulated in patients with adenocarcinoma, 19 of which were exclusively upregulated in patients without COPD. Patients with adenocarcinoma and COPD showed 12 additional deregulated miRNAs, nine of which were upregulated and three were downregulated. In patients with COPD only, seven miRNAs were found to be upregulated and seven downregulated. Additionally, the COPD and adenocarcinoma with COPD groups shared five deregulated miRNAs (two downregulated and three upregulated). Finally, the three pathological groups shared three upregulated miRNAs (miR-15b, miR-425 and miR-486-3p) as compared with the control group.

11 of these 66 differentially expressed miRNAs were randomly selected for validation in an independent cohort of patients. Results confirmed the miRNA expression profile obtained in the training set, achieving statistical significance for eight of them (miR-185, miR-192, miR-194, miR-195, miR-210, miR-365, miR451 and miR-486) (fig. 2).

In order to assess the potential functional interconnection of miRNAs discriminating each pathological entity studied, we investigated their chromosomal location. Four upregulated miRNA clusters located in distinct chromosomal regions (fig. 3) were identified in patients with adenocarcinoma and one in patients with COPD. The four upregulated genomic clusters in adenocarcinoma were the miR-17-92 miRNA cluster, located at chromosome locus 13q31.3, its two paralogues, the miR-106b-25 cluster (located on chromosome 7q22.1) and the miR-106a-363 cluster (located on the Xq26.2 chromosome), and the miR-192-194 cluster located on chromosome 11q13.1. The miR-17-92 cluster is composed of six members (miR-17, miR-18a, miR-19a, miR-19b, miR-20a and miR-92), all of which were found significantly overexpressed in the 
TABLE 1 Characteristics of the training cohort

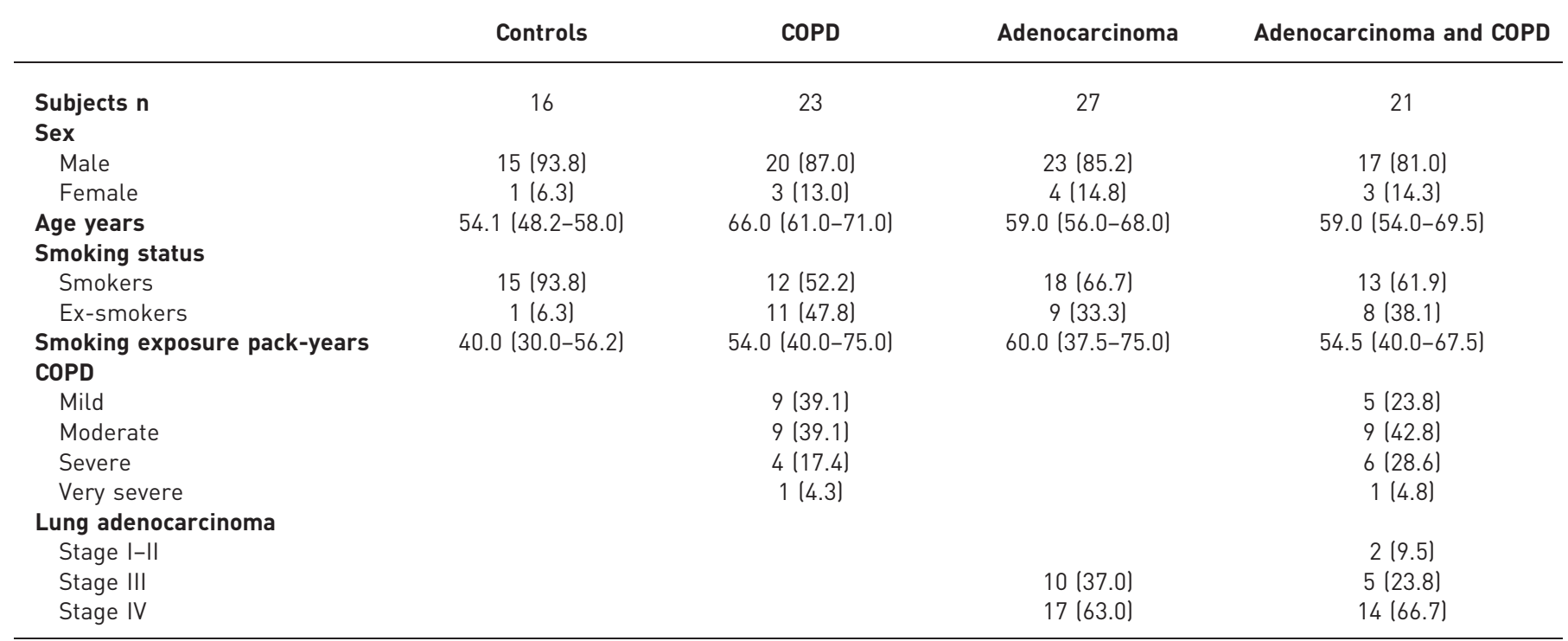

Data are presented as $\mathrm{n}(\%)$ or median (interquartile range), unless otherwise stated. Chronic obstructive pulmonary disease (COPD) severity was categorised according to the Global Initiative for Chronic Obstructive Lung Disease stages.

adenocarcinoma and adenocarcinoma with COPD groups (fig. 4 a-f). Similarly, five of the six miRNAs belonging to the miR-106a-363 cluster (miR-18, miR19b, miR20b, miR-106a, miR-92 and miR-363) and the three miRNAs belonging to the mir-106b-25 cluster (miR-106b, miR-93 and miR-25) were found to be upregulated in the adenocarcinoma (fig. $4 \mathrm{~g}-\mathrm{j}$ ) and adenocarcinoma with COPD groups (fig. 4k-m). Finally, expression levels of both members of the miR-192-194 cluster (miR-192 and miR-194) were also significantly increased in both groups with adenocarcinoma (fig. $4 n-0$ ). Regarding the group of patients with COPD, the miR-132-212 cluster located on chromosome $17 \mathrm{p} 13.3$ was found to be deregulated, with expression of both miRNAs in this cluster (miR-132 and miR-212) being significantly higher in COPD patients than in the control group (fig. 5).

\section{TABLE 2 Characteristics of the validation cohort}

\begin{tabular}{|c|c|c|c|c|}
\hline & Controls & COPD & Adenocarcinoma & Adenocarcinoma and COPD \\
\hline Subjects $\mathrm{n}$ & 10 & 10 & 10 & 10 \\
\hline \multicolumn{5}{|l|}{ Sex } \\
\hline Male & $6(60.0)$ & $10(100.0)$ & 9 (90.0) & $9(90.0)$ \\
\hline Female & $4(40.0)$ & & $1(10.0)$ & $1(10.0)$ \\
\hline Age years & $57.6(48.8-69.0)$ & $64.1(54.6-75.5)$ & $72.4(52.1-79.1)$ & $63.4(54.3-73.9)$ \\
\hline \multicolumn{5}{|l|}{ Smoking status } \\
\hline Smokers & $6(60.0)$ & $7(70.0)$ & $5(50.0)$ & $7(70.0)$ \\
\hline Ex-smokers & $4(40.0)$ & $3(30.0)$ & $5(50.0)$ & $3(30.0)$ \\
\hline Smoking exposure pack-years & $44.0(30.5-60.0)$ & $57.5(48.3-90.3)$ & $33.9(24.7-55.3)$ & $72.0(53.0-105.3)$ \\
\hline \multicolumn{5}{|l|}{ COPD } \\
\hline Mild & & 5 (50.0) & & $3(30.0)$ \\
\hline Moderate & & $4(40.0)$ & & $5(50.0)$ \\
\hline Severe & & $1(10.0)$ & & $2(20.0)$ \\
\hline \multicolumn{5}{|l|}{ Very severe } \\
\hline \multicolumn{5}{|l|}{ Lung adenocarcinoma } \\
\hline Stage I-II & & & $1(10.0)$ & $3(30.0)$ \\
\hline Stage III & & & & $4(40.0)$ \\
\hline Stage IV & & & $9(90.0)$ & $5(50.0)$ \\
\hline
\end{tabular}

Data are presented as $\mathrm{n}(\%)$ or median (interquartile range), unless otherwise stated. Chronic obstructive pulmonary disease (COPD) severity was categorised according to the Global Initiative for Chronic Obstructive Lung Disease stages. 


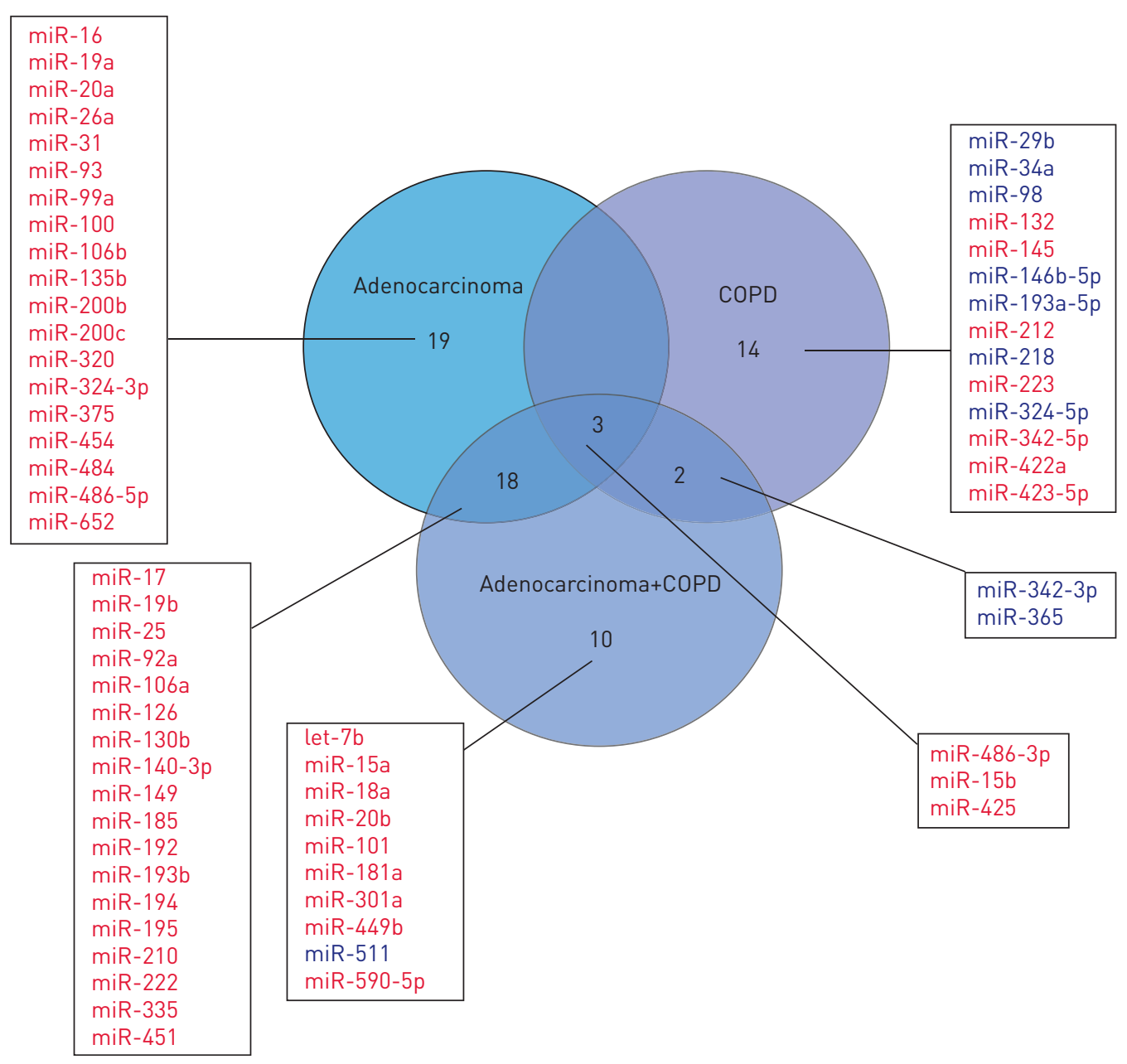

FIGURE 1 Venn diagram of microRNAs (miRNAs) associated with adenocarcinoma and/or chronic obstructive pulmonary disease (COPD). The lists show the miRNAs associated with each component of the diagram. miRNAs shown in red were upregulated and miRNAs shown in blue were downregulated.

Bioinformatic prediction analysis based on sequence similarity between miRNAs and mRNAs found that the miRNA clusters identified may potentially be involved in the regulation of several lung adenocarcinoma driver genes, such as AKT1, APC, ATM, CCND1, ERBB2, HRAS, KRAS, LKB1, MYC, NF1, PIK3CA, PTEN, RB1, TP53 and TERT. In addition, the miR-132-212 cluster associated with COPD may contribute to the regulation of functionally relevant genes in this chronic inflammatory lung disease, such as TLR2, TLR4, TLR5, MYC, MYD88, IRAK4, IL6, BDNF and AAT, among others. Most miRNA cluster-regulated genes have been experimentally confirmed by several authors (online supplementary table S1). Therefore, miRNA clusters might modulate these targets in a coordinated or individual manner, affecting several hallmarks of both diseases.

\section{Discussion}

In this study we have focused on assessing the role of miRNAs as potential biomarkers of two smoking-related diseases, adenocarcinoma and COPD, with the aim to discriminate commonly from selectively deregulated miRNAs associated with each pathological entity. Distinct miRNA expression profiles were indeed identified and validated for adenocarcinoma and COPD involving 66 differentially expressed miRNAs. Of note, this profiling was performed in a unique biological sample recovered from the BAL cells, which include primarily macrophages (80-90\%), lymphocytes $(<15 \%)$, erythrocytes and neutrophils $(2-5 \%)$, among other less frequently encountered cells. This model is a minimally invasive sample that is probably highly representative of the pulmonary airway niche. We believe these results are relevant and contribute to elucidating the distinct regulatory networks involved in the pathogenesis of adenocarcinoma and COPD, and may provide new tools of potential use as biomarkers for diagnosis and/or therapeutic purposes. 

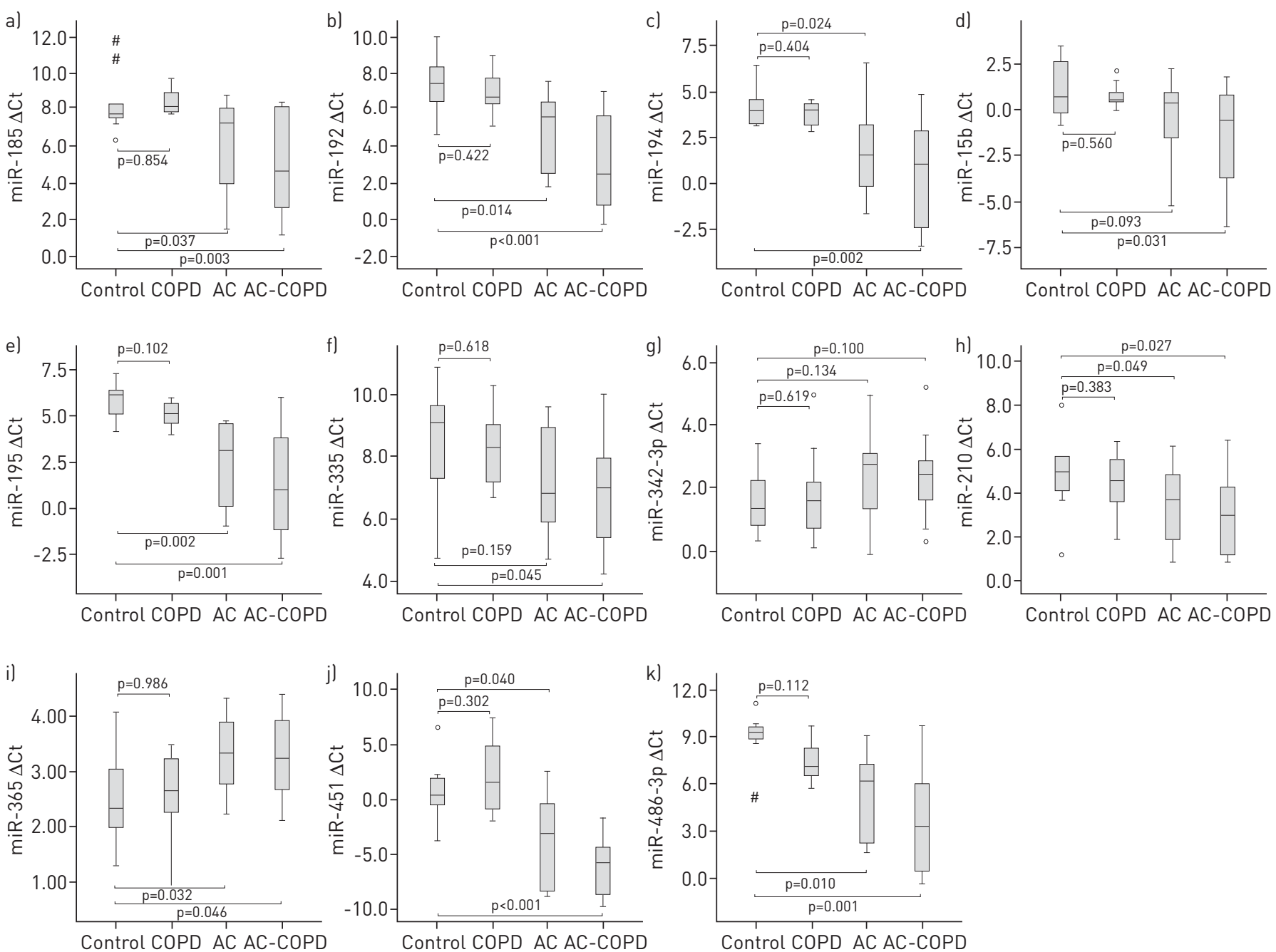

FIGURE 2 MicroRNA selection and validation by quantitative RT-PCR analysis in the validation cohort. a) miR-185, b) miR-192, c) miR-194, d) miR-15b, e) miR-195, f) miR335, g) miR-342-3p, h) miR-210, i) miR-365, j) miR-451 and k) miR-486-3p. Data are presented as $\Delta$ cycle threshold (Ct) values, with higher values indicating lower microRNA expression. Boxes show the interquartile range (IQR) and median (thick line); whiskers indicate the range. Outliers $>1.5 \times$ IQR but $<3 \times$ IQR from the nearest edge of the box are represented by open circles and those $>3 \times$ IQR from the nearest edge of the box are represented by ${ }^{\#}$ symbols. AC: adenocarcinoma; COPD: chronic obstructive pulmonary disease; AC-COPD: AC and COPD.

Overall, 66 miRNAs were found to be deregulated in one of the three pathological groups (adenocarcinoma, COPD, and adenocarcinoma with COPD) versus the control group (neither adenocarcinoma nor COPD). Consistent with our findings, two recent meta-analyses have reported 14 (miR-17, miR-18a, miR-20a, miR-20b, miR-31, miR-93, miR-106a, miR-130b, miR-135b, miR-193b, miR200b, miR-200c, miR-210 and miR-375) of the 52 upregulated miRNAs identified in our study among patients in the adenocarcinoma and adenocarcinoma with COPD groups to be overexpressed in human lung cancer tissue versus normal tissue (online supplementary table S2) [16, 17]. More specifically, studies profiling lung adenocarcinoma versus normal tissue have reported seven deregulated miRNAs, four upregulated (miR-21, miR-31, miR-182 and miR-210) and three downregulated (miR-126, miR-145 and miR-218) [18]. In our work, expression of miR-31 and miR-210 was also significantly higher among adenocarcinoma patients as previously described [18]. In line with these observations, the miR-210 has been recently proposed in combination with other four miRNAs for the early detection of nonsmall cell lung cancer [19].

Therefore, to date, there is convincing evidence supporting the potential role of miRNAs as biomarkers for lung cancer diagnosis and prognosis. However, further research is required to address their value as biomarkers in COPD. Few studies have investigated miRNA expression patterns associated with cigarette smoking and chronic inflammatory lung disease. In particular, the expression of let-7c, miR-1 and miR-146a was reported to be reduced in individuals with COPD compared with patients without COPD [20-22]. In addition, EzZIE et al. [23] found that the expression of miR-15b was upregulated in lung tissue from patients 


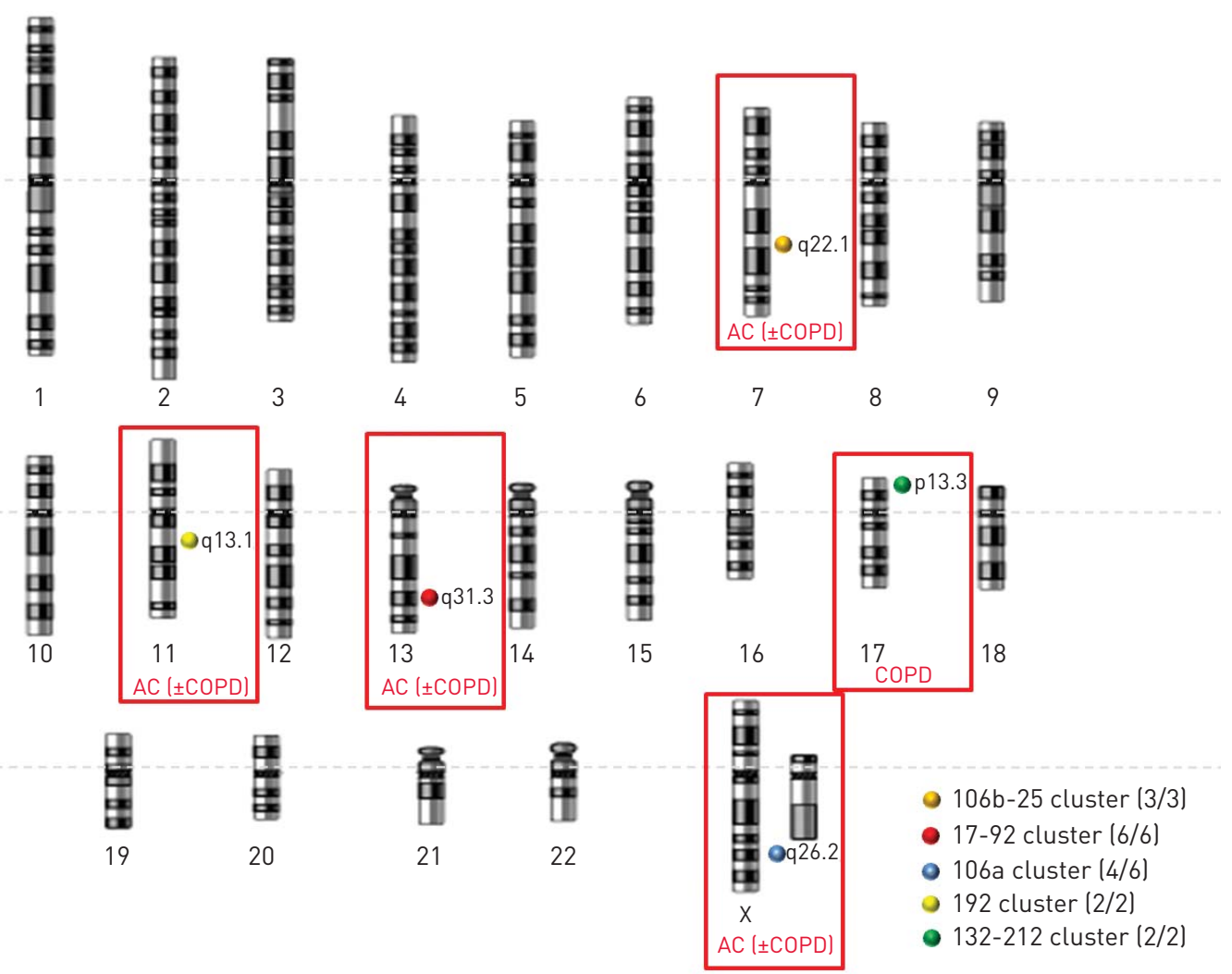

FIGURE 3 Chromosomal localisation of deregulated microRNA clusters in adenocarcinoma (AC) and chronic obstructive pulmonary disease (COPD). The members of the microRNA clusters that were differentially expressed are shown in brackets.

with COPD versus that from smokers without airway obstruction. In our study, we have identified nine downregulated miRNAs (miR-29, miR-34a, miR-98, miR-146b-5p, miR-193a-5p, miR-218, miR-324-5p, miR-342-3p and miR-365), while 10 were upregulated (miR-15b, miR-132, miR-145, miR-212, miR-223, miR-342-5p, miR-422a, miR-423-5p, miR-425 and miR-486-3p) in patients with COPD compared with the control group. Due to differences in the way the studies were designed, the results are expected to differ from one another. Moreover, the biological specimens employed for profiling have to be taken into account as it may significantly contribute to the widely variable results observed. In this regard it should be pointed out, as we have previously reported, that there is a very poor correlation between expression patterns observed in circulating plasma miRNAs compared to those assessed in BAL cell fraction-derived miRNAs from the same patient [11]. In our opinion, however, BAL fluid is likely more informative of the actual lung pathological conditions under study.

One potential limitation of our study is that concomitant medication was not registered or controlled for. Of particular relevance in this clinical scenario is the use of inhaled corticosteroids, common in patients with COPD and/or lung cancer, which may potentially affect miRNA expression levels. Nevertheless, the three pathological groups with increased likelihood to receive corticosteroid therapy only shared three upregulated miRNAs (miR-15b, miR-425 and miR-486-3p) compared to the control group. At present, to our knowledge, there are no studies that link the expression of these miRNAs with the effects of corticosteroids. In any case, it is important to highlight that our results clearly identified discriminating miRNA expression patterns for each pathological entity.

Once individual differentially expressed miRNAs were identified, we sought to investigate their potential functional network connection. miRNA gene clustering throughout the genome is significantly higher than would be expected at random, and miRNAs clusters are usually transcribed in a coordinated manner from common promoters. It has been proposed that miRNA clusters may be cooperatively suppressing targets through additive effects [7]. Hence, in our study, we analysed the chromosomal location and clustering of significantly deregulated miRNAs in each pathological condition studied. Overall, four miRNA clusters were identified as being upregulated in adenocarcinoma patients (miR-17-92, miR-106a-363, miR-106b-25 and miR-192-194) and one miRNA cluster (miR-132-212) in patients with COPD. It is interesting to note 

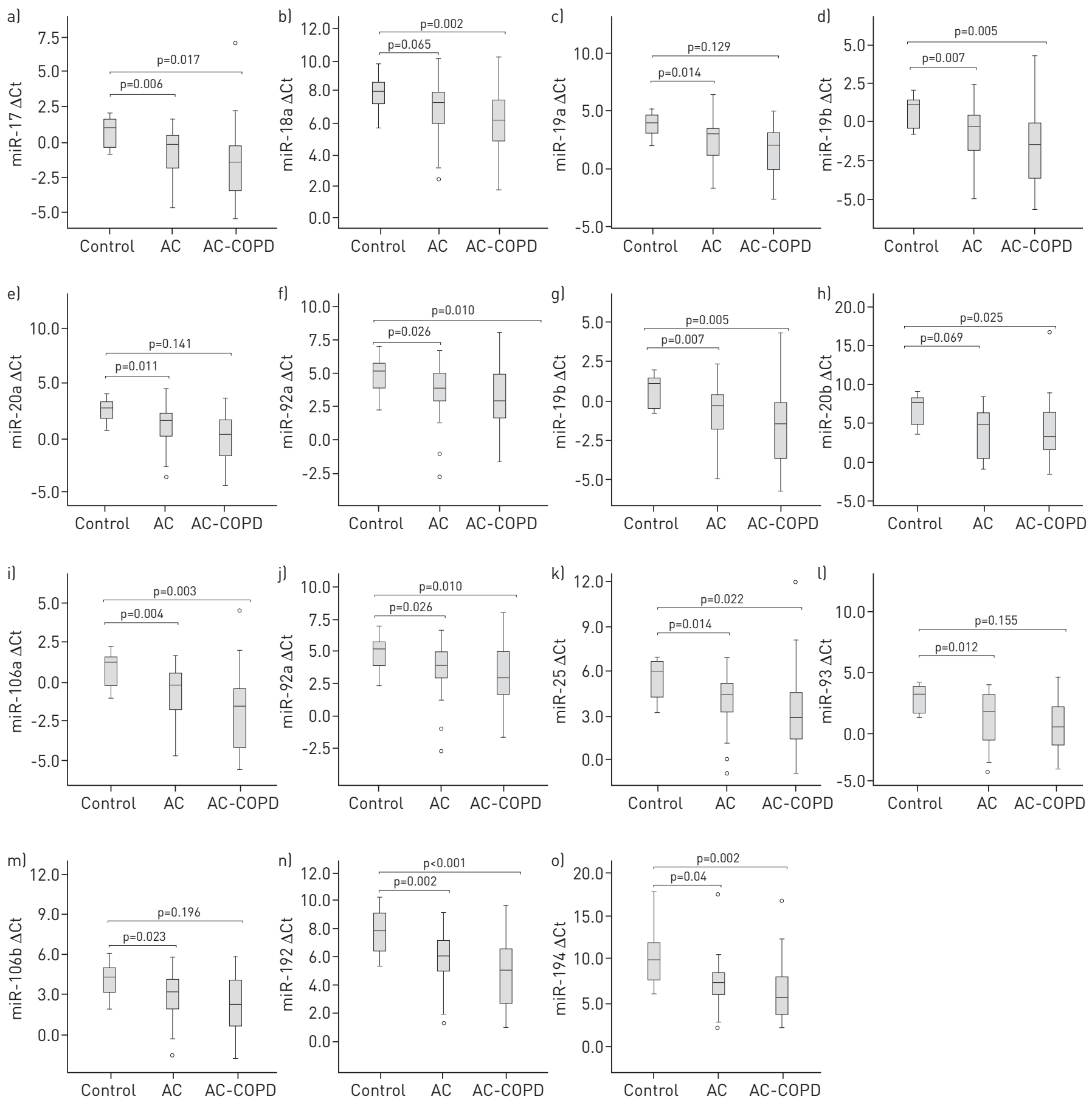

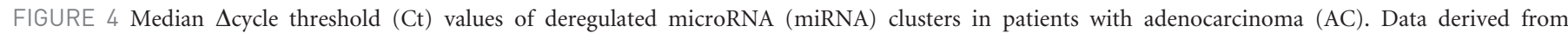

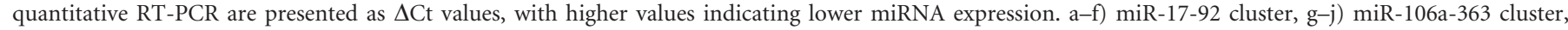

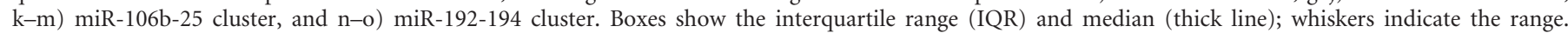

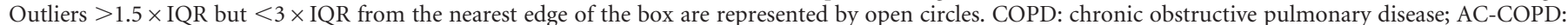
$\mathrm{AC}$ and COPD.

that the miR-17-92 cluster is frequently overexpressed in lung cancer cell lines, especially in those with small cell lung cancer histology [10]. RAPONI et al. [24] identified 15 miRNAs that were differentially expressed between normal lung and squamous cell lung carcinomas, including members of the miR-17-92 cluster and its paralogues, and found miRNA profiling of greater clinical utility in predicting the prognosis of patients with squamous cell lung carcinomas than mRNA-based signatures. Our study is the first to show an overexpression of the miR-17-92 cluster in patients with lung adenocarcinoma. Also, miR-17-92 paralogue 

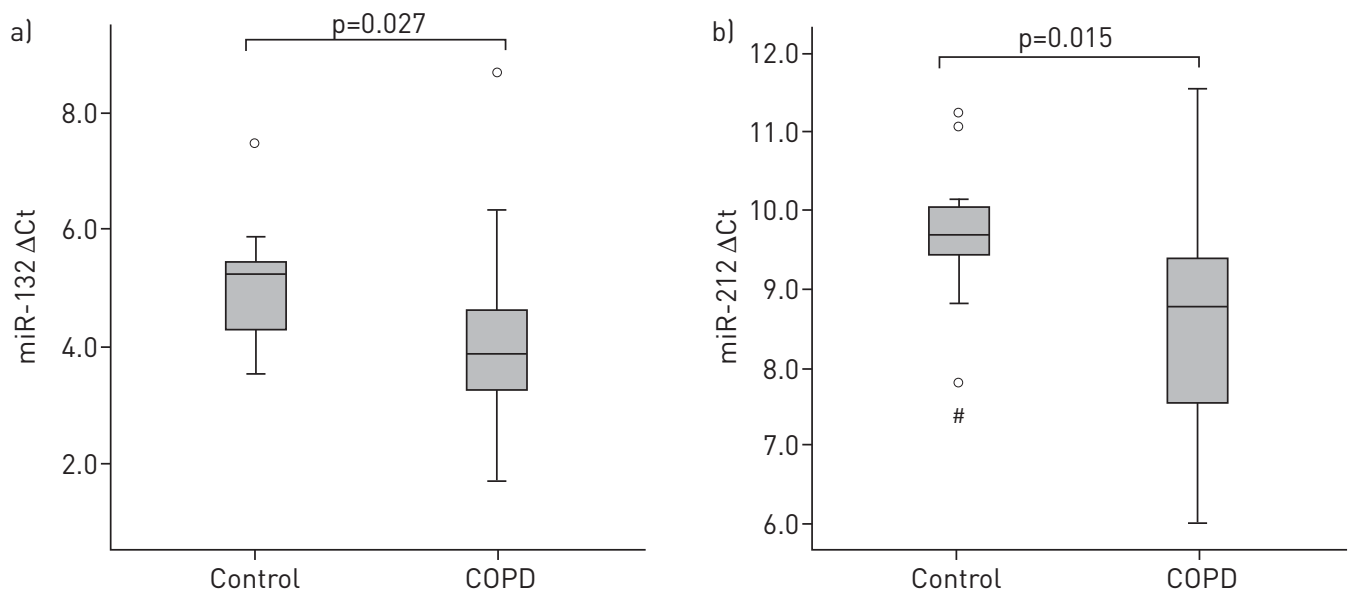

FIGURE 5 Median $\Delta$ cycle threshold (Ct) values of miR-132-212 cluster in chronic obstructive pulmonary disease (COPD). a) miR-132 and b) miR-212. Data derived from quantitative RT-PCR are presented as $\Delta$ Ct values, with higher values indicating lower microRNA expression. Boxes show the interquartile range (IQR) and median (thick line); whiskers indicate the range. Outliers $>1.5 \times \mathrm{IQR}$ but $<3 \times \mathrm{IQR}$ from the nearest edge of the box are represented by open circles and those $>3 \times \mathrm{IQR}$ from the nearest edge of the box are represented by ${ }^{\#}$ symbols.

clusters (miR-106a-363 and miR-106b-25) are co-expressed in these patients, suggesting related actions in this tumour type. Specifically, functional studies identified that upregulation of the miR-17-92 cluster is implicated in distinct pathways such as PI3K/AKT and transforming growth factor- $\beta$, among others $[25,26]$. In addition, the miR-192-194 cluster was significantly downregulated in lung adenocarcinoma. By contrast, the miR-132-212 cluster overexpressed in the COPD group, has been reported to be upregulated in alveolar macrophages of smokers compared to non-smokers [27]. However, no apparent correlation was observed in our study between the burden of cigarette smoking and expression patterns of miR-132 and miR-212 (data not shown). Indeed, although all our patients were active or ex-smokers, these miRNAs were not found to be deregulated among patients who developed adenocarcinoma, but rather exclusively among those with COPD only. Thus, the previously described upregulation of this miRNA cluster in smokers may be explained by the fact that tobacco is a risk factor for developing COPD, a condition that was not reported in the study mentioned. Consistent with our findings, $\alpha_{1}$-antitrypsin mRNA has been recently described as a true target for the miR-132-212 cluster [28], and its deficiency has been involved in COPD development [29]. We have found a negative correlation between $\alpha_{1}$-antitrypsin mRNA and miR-132-212 cluster expression (Supplementary figure 1).

In summary, the present study has identified distinct miRNA expression profiles in BAL fluid samples that are able to discriminate adenocarcinoma from COPD. Signatures showed common and specific deregulated miRNAs for each pathological condition, which will help unravel regulatory networks involved in the pathogenesis of these two smoking-related diseases and potentially provide new tools of clinical use as biomarkers for diagnosis and/or therapeutic purposes.

\section{References}

1 Young RP, Hopkins RJ, Christmas T, et al. COPD prevalence is increased in lung cancer, independent of age, sex and smoking history. Eur Respir J 2009; 34: 380-386.

2 de Torres JP, Marín JM, Casanova C, et al. Lung cancer in patients with chronic obstructive pulmonary disease incidence and predicting factors. Am J Respir Crit Care Med 2011; 184: 913-919.

3 Purdue MP, Gold L, Järvholm B, et al. Impaired lung function and lung cancer incidence in a cohort of Swedish construction workers. Thorax 2007; 62: 51-56.

Young RP, Hopkins RJ. COPD and lung cancer linked at a molecular genetic level. Chest 2011; 140: 266-267. Bentwich I, Avniel A, Karov Y, et al. Identification of hundreds of conserved and nonconserved human microRNAs. Nat Genet 2005; 37: 766-770.

Zhang B, Pan X, Cobb GP, et al. microRNAs as oncogenes and tumor suppressors. Dev Biol 2007; 302: 1-12.

Altuvia Y, Landgraf P, Lithwick G, et al. Clustering and conservation patterns of human microRNAs. Nucleic Acids Res 2005; 33: 2697-2706.

8 Landi MT, Zhao Y, Rotunno M, et al. MicroRNA expression differentiates histology and predicts survival of lung cancer. Clin Cancer Res 2010; 16: 430-441.

9 Yanaihara N, Caplen N, Bowman E, et al. Unique microRNA molecular profiles in lung cancer diagnosis and prognosis. Cancer Cell 2006; 9: 189-198.

10 Hayashita Y, Osada H, Tatematsu Y, et al. A polycistronic microRNA cluster, miR-17-92, is overexpressed in human lung cancers and enhances cell proliferation. Cancer Res 2005; 65: 9628-9632. 
11 Molina-Pinelo S, Suárez R, Pastor MD, et al. Association between the miRNA signatures in plasma and bronchoalveolar fluid in respiratory pathologies. Dis Markers 2012; 32: 221-230.

12 Applied Biosystems. User Bulletin \#2 ABI PRISM 7700 Sequence Detection System (P/N 4303859). http://www3. appliedbiosystems.com/cms/groups/mcb_support/documents/generaldocuments/cms_040980.pdf Date last accessed: March, 2014. Date last updated: October, 2001.

13 John B, Enright AJ, Aravin A, et al. Human MicroRNA targets. PLoS Biol 2004; 2: e363.

14 Lewis BP, Burge CB, Bartel DP. Conserved seed pairing, often flanked by adenosines, indicates that thousands of human genes are microRNA targets. Cell 2005; 120: 15-20.

15 Dweep H, Sticht C, Pandey P, et al. miRWalk - database: prediction of possible miRNA binding sites by "walking" the genes of three genomes. J Biomed Inform 2011; 44: 839-847.

16 Guan P, Yin Z, Li X, et al. Meta-analysis of human lung cancer microRNA expression profiling studies comparing cancer tissues with normal tissues. J Exp Clin Cancer Res 2012; 31: 54.

17 Võsa U, Vooder T, Kolde R, et al. Meta-analysis of microRNA expression in lung cancer. Int J Cancer 2013; 132: 2884-2893.

18 Yu L, Todd NW, Xing L, et al. Early detection of lung adenocarcinoma in sputum by a panel of microRNA markers. Int J Cancer 2010; 127: 2870-2878.

19 Roa WH, Kim JO, Razzak R, et al. Sputum microRNA profiling: a novel approach for the early detection of nonsmall cell lung cancer. Clin Invest Med 2012; 35: E271.

20 Van Pottelberge GR, Mestdagh P, Bracke KR, et al. MicroRNA expression in induced sputum of smokers and patients with chronic obstructive pulmonary disease. Am J Respir Crit Care Med 2011; 183: 898-906.

21 Sato T, Liu X, Nelson A, et al. Reduced miR-146a increases prostaglandin $\mathrm{E}_{2}$ in chronic obstructive pulmonary disease fibroblasts. Am J Respir Crit Care Med 2010; 182: 1020-1029.

22 Lewis A, Riddoch-Contreras J, Natanek SA, et al. Downregulation of the serum response factor/miR-1 axis in the quadriceps of patients with COPD. Thorax 2012; 67: 26-34.

23 Ezzie ME, Crawford M, Cho JH, et al. Gene expression networks in COPD: microRNA and mRNA regulation. Thorax 2012; 67: 122-131.

24 Raponi M, Dossey L, Jatkoe T, et al. MicroRNA classifiers for predicting prognosis of squamous cell lung cancer. Cancer Res 2009; 69: 5776-5783.

25 Rao E, Jiang C, Ji M, et al. The miRNA-17 92 cluster mediates chemoresistance and enhances tumor growth in mantle cell lymphoma via PI3K/AKT pathway activation. Leukemia 2012; 26: 1064-1072.

26 Dews M, Fox JL, Hultine S, et al. The myc-miR-17 92 axis blunts TGF $\beta$ signaling and production of multiple TGF $\beta$-dependent antiangiogenic factors. Cancer Res 2010; 70: 8233-8246.

27 Graff JW, Powers LS, Dickson AM, et al. Cigarette smoking decreases global microRNA expression in human alveolar macrophages. PLoS One 2012; 7: e44066.

28 Hassan T, Smith SG, Gaughan K, et al. Isolation and identification of cell-specific microRNAs targeting a messenger RNA using a biotinylated anti-sense oligonucleotide capture affinity technique. Nucleic Acids Res 2013; 41: e71.

29 Janciauskiene SM, Bals R, Koczulla R, et al. The discovery of $\alpha_{1}$-antitrypsin and its role in health and disease. Respir Med 2011; 105: 1129-1139. 